\title{
Peaked T-Waves and Potassium: The Reliability of Peaked T-Waves as Indicators of Hyperkalemia in End-Stage Renal Patients Undergoing Hemodialysis
}

\author{
Ryan D. Pappal*a, Ilse M. Espina ${ }^{b}$ Zubaid Rafique \\ ${ }^{a}$ Rice University, Houston, TX \\ ${ }^{b}$ Section of Emergency Medicine, Baylor College of Medicine, Houston, TX \\ Student:pappal.ryan@gmail.com*,pappal.ryan@rice.edu \\ Mentor:zubaidrafique@gmail.com*,rafique@bcm.edu
}

\begin{abstract}
While peaked T-waves have traditionally been associated with hyperkalemia cases, previous studies have called their reliability for hyperkalemia diagnosis into question. This study examined 89 ESRD patients totaling to 736 patient visits. Linear regressions and receiver operating characteristic (ROC) analyses were utilized to assess the reliability of peaked T-waves as a diagnostic criterion for hyperkalemia. Poor correlations between T/R ratio (leads V2-V4) and serum potassium were found, $\mathrm{r}=0.23$, 0.17 , and 0.17 , $p<0.001$. $\mathrm{R}^{2}$ values ranged from 0.03 to 0.05 . ROC analysis found poor sensitivity and specificity of $\mathrm{T} / \mathrm{R}$ as a predictor of hyperkalemia (area under the curve: $0.61-0.66$ ), and the $T / R \geq 0.75$ threshold yielded a sensitivity of 0.83 with a specificity of 0.40 when predicting hyperkalemia (serum potassium $>5.5 \mathrm{mEq} / \mathrm{L}$ ). We conclude that the peaked T-wave is a poor indicator for the early identification of hyperkalemia in ESRD patients presenting to the emergency department.
\end{abstract}

\section{KEYWORDS}

Hyperkalemia; Electrocardiography; End-stage Renal Disease; Hemodialysis; Arrhythmias, Potassium; Sensitivity and Specificity; ROC Curve

\section{INTRODUCTION}

Chronic kidney disease (CKD) is a common disorder that predisposes patients to dangerous metabolic conditions. ${ }^{1}$ Hypertension and diabetes mellitus are known risk factors for the development of CKD. The primary causes of renal failure are diabetic glomerulosclerosis and hypertensive nephrosclerosis, although chronic kidney disease has been known to precipitate from infections and exposure to illicit drugs and toxins, particularly in developing countries. ${ }^{2}$ Kidney dysfunction that progresses to the point that regular dialysis or a kidney transplant is required is defined as end-stage renal disease (ESRD). ${ }^{2}$

Hyperkalemia is defined as a serum potassium greater than $5.5 \mathrm{mEq} / \mathrm{L}$ and is a life-threatening electrolyte imbalance frequently observed in ESRD patients. ${ }^{1,3}$ In these patients, impaired glomerular filtration rate combined with the extracellular shift of potassium caused by acidosis impair the ability to excrete potassium. ${ }^{1,4}$ Hemodialysis is a common means for ESRD patients to maintain healthy electrolyte levels; however, at least one study has shown that the long hiatus from dialysis experienced during the weekend increases the risk of electrolyte imbalance formation, particularly hyperkalemia, and subsequently the risk of sudden cardiac death. ${ }^{3}$ Only modest changes in serum potassium in patients with existing cardiovascular or renal disease can lead to increased risk of hospitalization or death..$^{1,5}$

Hyperkalemia induces a wide range of vague symptoms, particularly muscle spasms, nausea, and vomiting. ${ }^{6}$ Additionally, it works through a number of mechanisms to impair myocardial electrical conduction. Mild hyperkalemia, serum potassium between 5.5 $6.5 \mathrm{mEq} / \mathrm{L},{ }^{7}$ inhibits voltage-gated sodium channels by reducing the resting membrane potential. The subsequent decrease in $\mathrm{V}_{\max }$ of these sodium channels blunts the rate of depolarization during phase 0 of the action potential, causing P-wave and QRS duration prolongation. ${ }^{7}$ When serum potassium rises above $6.5 \mathrm{mEq} / \mathrm{L},{ }^{7} \mathrm{IKr}$-mediated potassium currents increase due to heightened channel sensitivity from high extracellular potassium. Subsequent membrane repolarization during phase 3 of the myocardial action potential occurs more rapidly and thus with greater magnitude, manifesting as short-duration, peaked T-waves on ECG. ${ }^{7}$ Figure 1 shows a hyperkalemic patient's ECG with peaked T-waves and a prolonged QRS interval (A), as well as one from a different hyperkalemic patient that has normal T-waves and a standard QRS interval (B). As serum potassium progresses to well above $8.0 \mathrm{mEq} / \mathrm{L}$, the QRS complex widens substantially and can fuse with the T-wave, forming a classic sine-wave ECG pattern that is indicative of impending cardiac arrest from ventricular fibrillation or asystole. ${ }^{6,8,9}$ 


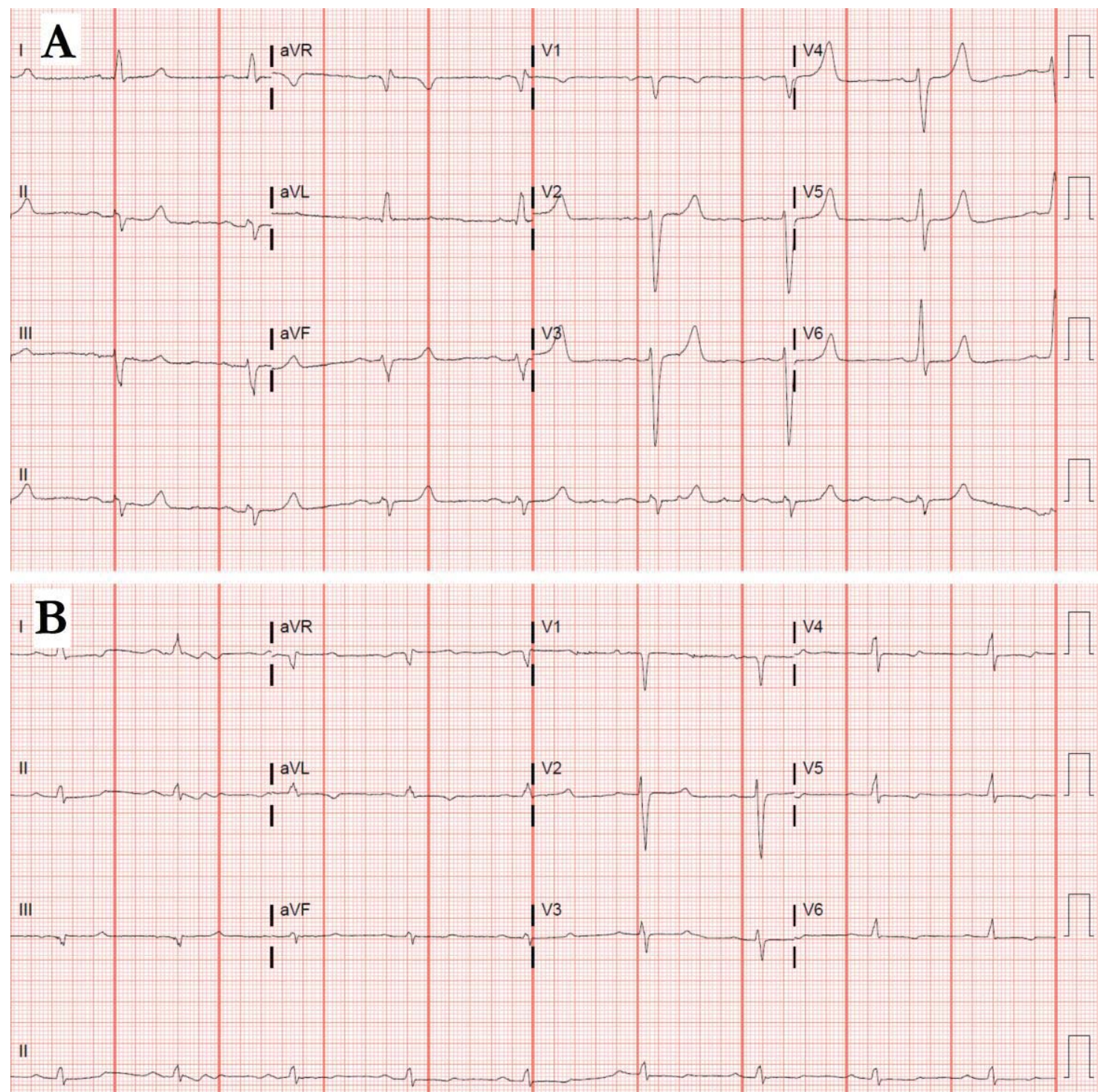

Figure 1. ECGs from ESRD patients in severe hyperkalemia. A) Patient with a serum potassium of $7.3 \mathrm{mEq} / \mathrm{L}$. ECG shows peaked Twaves in V2, V3, V4 and QRS prolongation (QRSD = $138 \mathrm{~ms}$ ). B) Patient with a serum potassium of $7.5 \mathrm{mEq} / \mathrm{L}$. ECG has non-peaked Twaves and normal QRS duration $(\mathrm{QRSD}=104 \mathrm{~ms})$.

Since hyperkalemia may cause serious cardiac complications, rapid identification and treatment are essential to improving outcomes. Clinical guidelines suggest the utilization of 12-lead electrocardiograms (ECGs) to detect hyperkalemia alongside blood tests. ${ }^{9}$ However, the status of peaked T-waves as indicators of hyperkalemia has been called into question in some recent retrospective studies. A review of 127 instances of diagnosed hyperkalemia showed that only $46 \%$ had any ECG changes suggestive of hyperkalemia; furthermore, the severity of hyperkalemia had no significant correlation with the presence of classic ECG signs. ${ }^{10} \mathrm{~A}$ study of 74 hemodialysis patients reported no significant differences in $\mathrm{T}$-wave amplitudes and $\mathrm{T} / \mathrm{R}$ ratios in the precordial leads between normal and elevated serum potassium level.11 In another review of 90 hyperkalemic patients, only 24 were noted to have T-wave abnormalities, with 21 of those noted as non-specific changes by reading cardiologists; furthermore, only 47 of the 90 patients had any ECG changes at all. ${ }^{6}$

The diagnostic difficulties caused by the unreliability of classic hyperkalemic ECG changes are exacerbated by the tendency of hyperkalemia to cause other, non-specific cardiac abnormalities. Severe hyperkalemia can also manifest in heart blocks, including fascicular blocks, bundle branch blocks, and complete heart block. ${ }^{13}$ The effect of hyperkalemia on ventricular repolarization has been known to cause widespread ST-elevation mimicking acute myocardial infarction patterns. ${ }^{6,13}$ Thus, even when hyperkalemia is manifesting in ECG changes, a clinician must be ready to screen out extraneous changes that suggest other dangerous 
conditions before chiefly suspecting hyperkalemia. Thus, if peaked T-waves are to be utilized as a diagnostic criterion, favorable sensitivity and specificity for ECG-based parameters must be established.

Our study examines ECG and electrolyte data from 89 ESRD patients who have undergone hemodialysis for at least three months. We examined whether or not peaked T-waves are reliable indicators of hyperkalemia in ESRD patients.

\section{METHODS AND PROCEDURES}

This study was a retrospective chart review of a cohort of dialysis patients at an inner city county hospital in a major metropolitan city. Eighty-nine ESRD patients were included in the review who visited Ben Taub General Hospital between June 2012 and June 2014. The study was approved by the Baylor College of Medicine institutional review board. Patients were included if they were older than 18 years of age and received dialysis for more than 3 months. Patients were excluded if they did not have a readable ECG tracing or a recorded basic metabolic panel associated with their tracing.

A total of 89 patients (49 male) were enrolled and 736 data points were recorded. Each data point was a unique patient-visit for emergent dialysis, consisting of one ECG and corresponding electrolyte levels. ECGs used were from intake before any treatment was administered. Peaked $\mathrm{T}$-wave definition of $\mathrm{T} / \mathrm{R}$ ratio $\geq 0.75$ was used to calculate its prevalence. $\mathrm{T}$-wave and $\mathrm{R}$-wave amplitudes were measured by hand on printed ECGs for leads V2, V3, and V4. ECG data was measured without knowledge of serum potassium level.

Data points were excluded if the electronic interpretation of the ECGs included left ventricular hypertrophy, right or left bundle branch block, or artificial pacemaker spikes. Furthermore, ECGs with a wandering baseline or other characteristic that prevented a reliable measurement of $\mathrm{T} / \mathrm{R}$ were excluded. Unreadable $\mathrm{R}$-wave amplitudes due to low voltage were also excluded. To assess our hypothesis that peaked $\mathrm{T}$-waves are poor clinical indicators of hyperkalemia, we assessed for an association between $\mathrm{T} / \mathrm{R}$ ratio and serum potassium level with linear regression models in leads V2, V3, and V4, and an interaction with calcium level was assessed for. Then, to determine the how well peaked T-wave performed as a diagnostic criterion for hyperkalemia, we ran receiver operating characteristic analyses. Custom statistical packages (PSPP and R) and plotting software (Microsoft Excel) were utilized for analysis. The specialized R package ROCR was utilized for receiver operating characteristic visualization and analysis. ${ }^{12}$

\begin{tabular}{ll}
\hline Variable & Value \\
\hline Patients & 89 \\
Mean Age \pm S.D. & $46 \pm 15$ \\
Sex & \\
Male & 49 \\
Female & 40 \\
Race (Ethnicity) & 85 \\
Hispanic / Latino & 2 \\
Black / African American & 2 \\
Middle Eastern & 736 \\
Patient-Visits & 547 \\
Hyperkalemia & $358-65.4 \%$ \\
Peaked T-Wave & $189-34.6 \%$ \\
Normal T-Wave & 189 \\
Normokalemia & $80-42.3 \%$ \\
Peaked T-Wave & $109-57.7 \%$ \\
$\quad$ Normal T-Wave & \\
Mean Electrolyte Levels \pm S.D. & $5.91 \pm 0.90$ \\
Potassium & $107.1 \pm 5.39$ \\
Chloride & $93.8 \pm 26.8$ \\
Blood Urea Nitrogen & $13.2 \pm 6.03$ \\
Creatinine & $0.96 \pm 0.15$ \\
Ionized Calcium & \\
\hline Table 1. Demographics, prevalence, and descriptive statistics. \\
aHyperkalemia is defined as serum potassium $\geq 5.5 \mathrm{mEq} / \mathrm{L}$ \\
bPeaked T-wave is defined as T/R ratio $\geq 0.75$ in V2, V3, or V4 \\
\hline
\end{tabular}




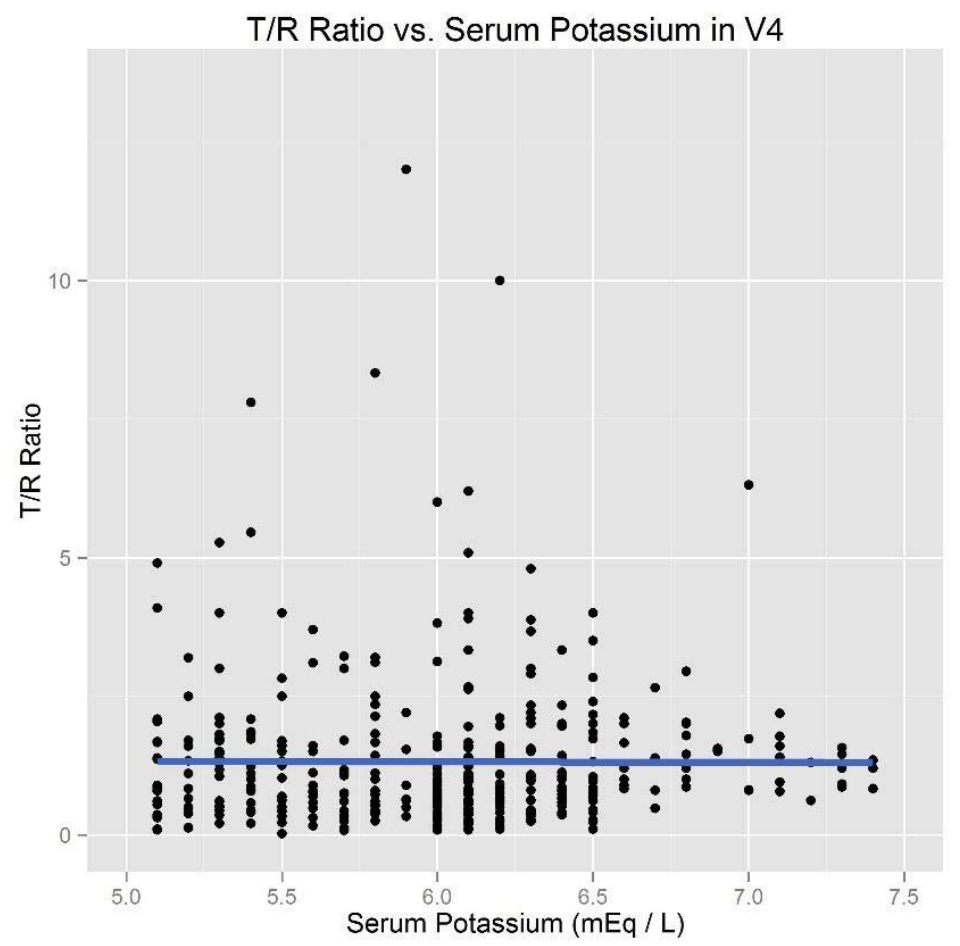

Figure 2. Linear regression model in V4. Graph shows T-wave to R-wave amplitude ratio vs. serum potassium scatter plot with a regression line. Analysis revealed a poor correlation consistent with that in leads V2 and V3, $\mathrm{r}=0.17, p<0.001$. Only $3 \%$ of the variation in $\mathrm{T} / \mathrm{R}$ ratio data could be explained by the linear model, $\mathrm{R}^{2}=0.03$.

\section{RESULTS}

The mean potassium level $\left(\left[\mathrm{K}^{+}\right]\right)$for the data set was $5.9( \pm 0.9) \mathrm{mEq} / \mathrm{L}$, and $\left[\mathrm{K}^{+}\right]$ranged from $3.1-8.4 \mathrm{mEq} / \mathrm{L}$. There were 49 males (55\%) and 40 females (45\%) in the cohort. The sample overwhelmingly identified as Hispanic/Latino (96\%). The mean age of the participants was 46.6 ( \pm 15.1 ) years. The prevalence of acute hyperkalemia was $74 \%$. Using the definition of a peaked $\mathrm{T}$ wave as $\mathrm{T} / \mathrm{R} \geq 0.75$, the prevalence of peaked $\mathrm{T}$-wave appearance in any of V2, V3, or V4 was 59.5\%. Specific prevalence of peaked T-waves in V2, V3, and V4 were $48.0 \%, 39.0 \%$, and $21.1 \%$ respectively. Demographics of participants and descriptive statistics for collected data is summarized in Table 1.

\begin{tabular}{lll}
\hline Lead & Correlation Coefficient $(\mathbf{r})$ & Adjusted $\mathbf{R}^{2}$ \\
\hline V2 & $0.23^{*}$ & 0.05 \\
V3 & $0.17^{*}$ & 0.03 \\
V4 & $0.17^{*}$ & 0.03 \\
\hline
\end{tabular}

Table 2. Statistical correlation of $\mathbf{T} / \mathbf{R}$ ratio vs. serum potassium, by lead. A multiple regression analysis on $\mathrm{T}$-wave to $\mathrm{R}$-wave amplitude ratios vs. serum potassium levels revealed poor correlations. Correlation coefficients ranged from $0.17-0.24$, showing weak effects of serum potassium on $\mathrm{T} / \mathrm{R}$ ratio. All adjusted $\mathrm{R}^{2}$ values are at or under 0.05 , indicating that no more than $5 \%$ of the variability in $\mathrm{T}$-wave amplitudes or $\mathrm{T} / \mathrm{R}$ ratios could be explained by serum potassium levels. ${ }^{*} p<0.001$

Linear correlation data is summarized in Table 2. Regression analysis of $\mathrm{T} / \mathrm{R}$ ratio vs. serum potassium for V2, V3, and V4, yielded poor correlations, with coefficients of $\mathrm{r}=0.23, \mathrm{r}=0.17$, and $\mathrm{r}=0.17$, respectively. While all correlations were statistically significant, $p<0.001$, variance accounted for by these models was limited, with $\mathrm{R}^{2}$ values of 0.05 , 0.03 , and 0.03 , respectively. No more than $5 \%$ of the variance in $\mathrm{T} / \mathrm{R}$ ratio was accounted for by the linear model with serum potassium. Scatter plot showing $\mathrm{T} / \mathrm{R}$ ratio vs. serum potassium for V4 is shown in Figure 2. The linear partial correlation was run while controlling for serum calcium. No changes in correlation significance or effect size were observed (data not shown). 


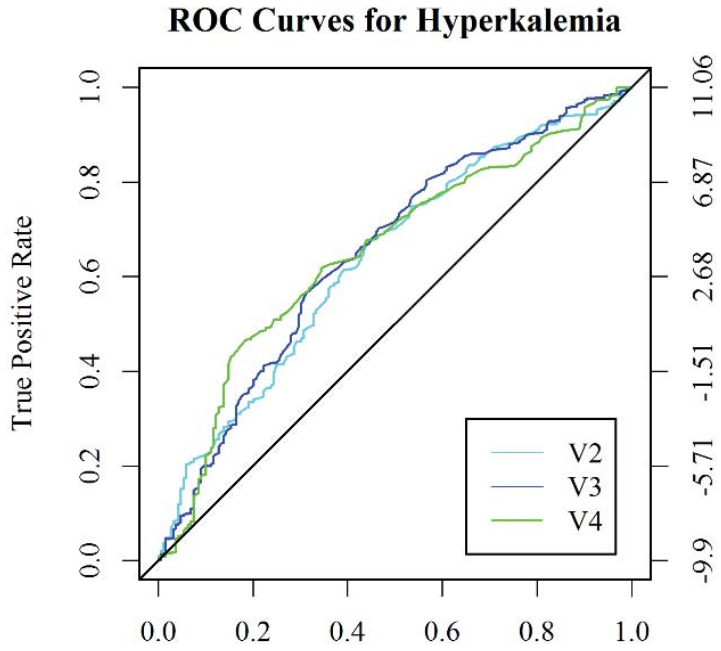

False Positive Rate
ROC Curves for Severe Hyperkalemia

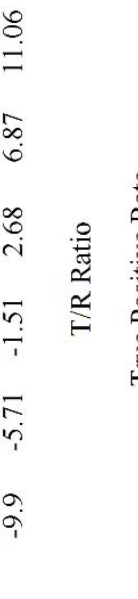

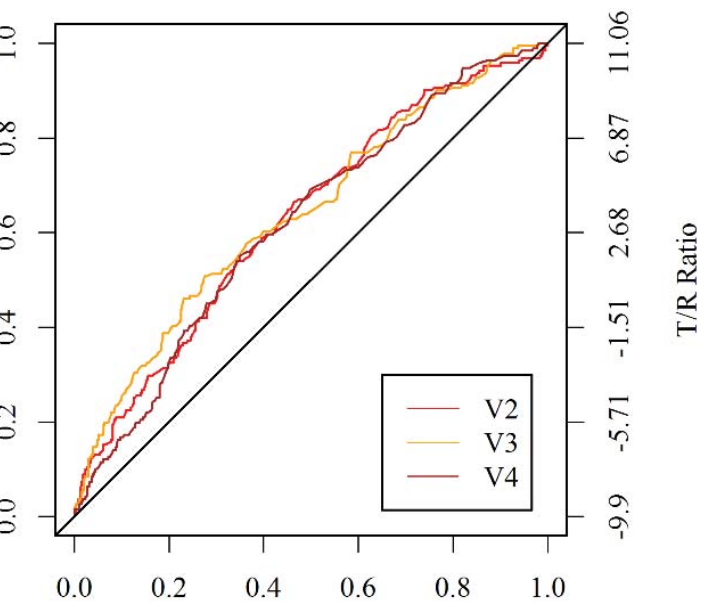

False Positive Rate

Figure 3. ROC curve analysis. Left-hand graph shows ROC curves for $\mathrm{T} / \mathrm{R}$ ratio as a diagnostic criterion for hyperkalemia (serum potassium $\geq 5.5 \mathrm{mEq} / \mathrm{L}$ ). Right-hand graph shows ROC curves for $\mathrm{T} / \mathrm{R}$ ratio as a diagnostic criterion for severe hyperkalemia (serum potassium $\geq 6.5 \mathrm{mEq} / \mathrm{L}$ ). The area under the curve (AUC) was calculated for each curve. All six ROC curves had AUCs significantly higher than the diagonal reference line with AUC $=0.5, p<0.001$. AUCs for the curves ranged from 0.61 to 0.66 .

Sensitivity and specificity were calculated for the peaked $T$-wave cutoff of $T / R \geq 0.75$. For hyperkalemia $\left(\left[\mathrm{K}^{+}\right] \geq 5.5\right.$ $\mathrm{mEq} / \mathrm{L})$, sensitivity and specificity were 0.83 and 0.40 , respectively. For severe hyperkalemia $\left(\left[\mathrm{K}^{+}\right] \geq 6.5 \mathrm{mEq} / \mathrm{L}\right)$, sensitivity and specificity were 0.88 and 0.27 , respectively. Receiver operating characteristic (ROC) analysis was utilized with $\mathrm{T} / \mathrm{R}$ ratio as a predictor of hyperkalemia (serum potassium $\geq 5.5 \mathrm{mEq} / \mathrm{L}$ ) and severe hyperkalemia (serum potassium $\geq 6.5 \mathrm{mEq} / \mathrm{L}$ ). Optimal $\mathrm{T} / \mathrm{R}$ ratio predictive thresholds were calculated by finding the maximum summed square of sensitivity and specificity. The area under the curve (AUC) was computed. The ROC curves are presented in Figure 3. Results of the analysis are summarized in Table 3. Optimal $\mathrm{T} / \mathrm{R}$ ratio predictive thresholds for hyperkalemia in $\mathrm{V} 2$, V3, and V4 were $0.88,0.81$, and 0.42 , respectively. Sensitivities (and specificities) for these thresholds were $0.61(0.61), 0.59(0.66)$, and 0.62 (0.66), respectively. Computed AUCs of the ROC curves for $\mathrm{T} / \mathrm{R}$ ratio for both hyperkalemia and severe hyperkalemia ranged from 0.60 to $0.66, p<0.001$.

\begin{tabular}{|c|c|c|c|c|c|}
\hline & Lead & Optimal T/R Threshold & Optimal Sensitivity & Optimal Specificity & $\begin{array}{l}\text { Area Under ROC Curve } \\
(95 \% \mathrm{CI})\end{array}$ \\
\hline \multirow{3}{*}{ 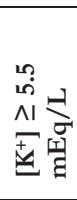 } & V2 & 0.88 & 0.614 & 0.608 & $\begin{array}{l}0.638^{*} \\
(0.592-0.684)\end{array}$ \\
\hline & V3 & 0.81 & 0.592 & 0.656 & $\begin{array}{l}0.651^{*} \\
(0.605-0.697)\end{array}$ \\
\hline & V4 & 0.42 & 0.618 & 0.656 & $\begin{array}{l}0.652^{*} \\
(0.608-0.697)\end{array}$ \\
\hline \multirow{3}{*}{ 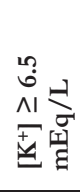 } & V2 & 1.11 & 0.586 & 0.606 & $\begin{array}{l}0.629 * \\
(0.584-0.675)\end{array}$ \\
\hline & V3 & 0.99 & 0.576 & 0.637 & $\begin{array}{l}0.640^{*} \\
(0.594-0.686)\end{array}$ \\
\hline & V4 & 0.61 & 0.550 & 0.651 & $\begin{array}{l}0.619 * \\
(0.573-0.664)\end{array}$ \\
\hline
\end{tabular}

Table 3. Summary of receiver operating characteristic analysis on $\mathbf{T} / \mathbf{R}$ ratio. Receiver operating characteristic (ROC) analysis was run on $\mathrm{T} / \mathrm{R}$ ratio for predictive performance of hyperkalemia (serum potassium $\geq 5.5 \mathrm{mEq} / \mathrm{L}$ ) and severe hyperkalemia (serum potassium $\geq 6.5 \mathrm{mEq} / \mathrm{L}$ ). An optimal T/R ratio threshold was determined for each lead by optimizing true positive rate while minimizing false positive rate. These thresholds are reported above with the corresponding sensitivity and specificity values. The area under each ROC curve was computed and revealed poor criterion performance, with all calculated areas between 0.61 and $0.66 .{ }^{*} p<0.001$ 


\section{DISCUSSION}

Our study examined 89 end-stage renal disease patients receiving emergent dialysis for at least three months. $\mathrm{T} / \mathrm{R}$ ratio has statistically significant but clinically poor correlation with serum potassium. The optimal correlation was noted in lead V2, where still only $5 \%$ of the variation in $\mathrm{T} / \mathrm{R}$ ratio was accounted for by serum potassium. Furthermore, receiver operating characteristic (ROC) analysis on $\mathrm{T} / \mathrm{R}$ ratio in $\mathrm{V} 2, \mathrm{~V} 3$, and $\mathrm{V} 4$ as predictive of hyperkalemia $\left(\left[\mathrm{K}^{+}\right] \geq 5.5 \mathrm{mEq} / \mathrm{L}\right)$ and severe hyperkalemia $\left(\left[\mathrm{K}^{+}\right]\right.$ $\geq 6.5 \mathrm{mEq} / \mathrm{L}$ ) indicated poor diagnostic performance. Optimal thresholds calculated by greatest summed square of sensitivity and specificity also yielded poor performance, with sensitivities ranging from 0.55 to 0.62 , and specificities ranging from 0.61 to 0.66 . We conclude that $\mathrm{T} / \mathrm{R}$ ratios have poor predictive value for the occurrence of hyperkalemia in ESRD patients.

Our results largely replicate past studies showing poor sensitivity and specificity of peaked T-wave presentation in hyperkalemic patients. ${ }^{6,10,11}$ Aslam et al. found that serum calcium had was a significant variable when using a linear model on T-wave height in ESRD patients. ${ }^{11}$ Our study contradicts this and found that when added as a variable, serum calcium did not affect the correlation of the linear model compared to serum potassium alone. Wrenn et al. found that sensitivity and specificity of the peaked T-wave, as determined by physician readers, mildly improved when predicting severe hyperkalemia $\left(\left[\mathrm{K}^{+}\right]>6.5 \mathrm{mEq} / \mathrm{L}\right)$ compared to hyperkalemia $\left(\left[\mathrm{K}^{+}\right]>5.5 \mathrm{mEq} / \mathrm{L}\right) .{ }^{13}$ We ran two separate ROC analyses for these two serum potassium cutoffs. Our results contradicted Wrenn et al., finding that criterion performance did not significantly change between the two potassium cutoffs.

Our study has several limitations. As this was a retrospective study, several general biases may have affected our conclusions. First, hospital protocol dictated that 12-lead ECGs were taken on those suspected of cardiac ischemia or hyperkalemia, limiting the availability of healthy controls. Additionally, hospital lab protocol did not screen blood samples for falsely elevated potassium levels from hemolysis; however, this was considered insignificant because treating physicians trusted this lab data in clinical decision-making. This study was specific to ESRD patients and does not provide evidence for or against the use of peaked Twaves as a diagnostic criterion for hyperkalemia in patients with normal kidney function. The participants in this study were overwhelmingly Hispanic/Latino, and caution should be taken in applying these results to all races and ethnicities. Additionally, this study examined only the T-wave characteristic on ECG. Future studies should incorporate other variables such as PR interval, QRS duration, and QTc interval, which can be altered by potassium imbalances. ${ }^{7,8}$

\section{CONCLUSIONS}

T/R ratios show poor correlation with patients' potassium levels, and peaked T-waves are poor clinical indicators of hyperkalemia in ESRD patients presenting to the emergency department.

\section{ACKNOWLEDGMENTS}

The authors thank Lisa Basgall for her maintenance of the Rice EMS Research Course. The authors also thank Kelly Keene Rogers for her work organizing and maintaining the research project. 


\section{REFERENCES}

1. Einhorn, L. M., Zhan, M., Hsu, V. D., Walker, L. D., Moen, M. F., Seliger, S. L., Weir, M. R., Fink, J. C. The frequency of hyperkalemia and its significance in chronic kidney disease. Arch Intern Med. 2009;169(12):1156-1162. doi:10.1001/archinternmed.2009.132.

2. Levey, A. S., Coresh, J. Chronic kidney disease. Lancet. 2012;379(9811):165-180. doi:10.1016/S0140-6736(11)60178-5.

3. Dillon, J. J., DeSimone, C. V., Sapir, Y., Somers, V. K., Dugan, J. L., Bruce, C. J., Ackerman, M. J., Asirvatham, S. J., Striemer, B. L., Bukartyk, J., Scott, C. G., Bennet, K. E., Mikell, S. B., Ladewig, D. J., Gilles, E. J., Geva, A., Sadot, D., Friedman, P. A. Noninvasive potassium determination using a mathematically processed ECG: Proof of concept for a novel "blood-less, blood test." J Electrocardiol. 2015;48(1):12-18. doi:10.1016/j.jelectrocard.2014.10.002.

4. Hollander-Rodriguez, J. C., Calvert, J. F. Hyperkalemia. Am Fam Physician. 2006;73(2):283-290.

5. Jain, N., Kotla, S., Little, B. B., Weideman, R. A., Brilakis, E. S., Reilly, R. F, Banerjee, S. Predictors of hyperkalemia and death in patients with cardiac and renal disease. Am J Cardiol. 2012;109(10):1510-1513. doi:10.1016/j.amjcard.2012.01.367.

6. Montague, B. T., Ouellette, J. R., Buller, G. K. Retrospective review of the frequency of ECG changes in hyperkalemia. Clin J Am Soc Nephrol. 2008;3(2):324-330. doi:10.2215/CJN.04611007.

7. Parham, W. A., Mehdirad, A. A., Biermann, K. M., Fredman, C. S. Hyperkalemia revisited. Tex Heart Inst J. 2006;33(1):4047.

8. Levis, J. T. ECG Diagnosis: Hyperkalemia. Perm Journal. 2013;17(1):69.

9. Martinez-Vea, A., Bardají, A., Garcia, C., Oliver, J. A. Severe hyperkalemia with minimal electrocardiographic manifestations: a report of seven cases. J Electrocardiol. 1999;32(1):45-49. doi:10.1016/S0022-0736(99)90020-1.

10. Acker, C., Johnson, J., Palevsky, P., Greenberg, A. Hyperkalemia in hospitalized patients: causes, adequacy of treatment, and results of an attempt to improve physician compliance with published therapy guidelines. Arch Intern Med. 1998;158:917924. doi:10.1023/A:1007135517950.

11. Aslam, S., Friedman, E. A., Ifudu, O. Electrocardiography is unreliable in detecting potentially lethal hyperkalaemia in haemodialysis patients. Nephrol Dial Transplant. 2002;17(9):1639-1642. doi:10.1093/ndt/17.9.1639.

12. Sing, T., Sander, O., Beerenwinkel, N., Lengauer, T. ROCR: Visualizing classifier performance in R. Bioinformatics. 2005;21(20):3940-3941. doi:10.1093/bioinformatics/bti623.

13. Wrenn, K. D., Slovis, B. S., Slovis, C. M. The ability of physicians to predict electrolyte deficiency from the ECG. Ann Emerg Med. 1990;19(5):580-583. doi:10.1097/00132586-199212000-00002.

\section{STUDENT AUTHOR}

Ryan Pappal is a fourth-year undergraduate student at Rice University in Houston, TX. A pre-medical student and certified paramedic, Ryan is pursuing degrees in Biochemistry \& Cell Biology and Psychology in preparation for medical school. He will graduate Rice in May 2017.

\section{PRESS RELEASE}

High potassium levels, called hyperkalemia, can cause serious illness in patients. Because kidney disease patients develop hyperkalemia from their disease, we investigated a possible diagnostic criterion from electrocardiograms, called the peaked $\mathrm{T}$ wave, which has long been associated with hyperkalemia. Our results found that the presence or absence of peaked T-waves on electrocardiograms alone are not sufficient to predict whether these kidney disease patients have hyperkalemia or not. Other diagnostic criteria should be developed for the better diagnosis of these patients. 